\title{
Programación Neurolingüística como estrategia en el desempeño académico de los estudiantes de la Facultad de Ciencias Básicas y de la educación. UPC
}

\section{Neurro-linguistic programming as a strategy in the academic performance of students of the Faculty of Basic Sciences and Education. UPC}

\section{${ }^{1} \mathrm{Br}$. Jhon Arjona De León}

${ }^{2} \mathrm{Br}$. Eva De la Hoz Contreras

Universidad del Cesar

${ }^{3}$ Dra. María Mercedes Colina

Universidad Mariana

\section{${ }^{4}$ Dr. Teovaldo García Romero}

Universidad Popular del Cesar

\section{RESUMEN}

El objetivo de la investigación es analizar la programación neurolingüística como estrategia en el desempeño académico de los estudiantes de la Facultad de Ciencias Básicas y de la Educación. Las variables de estudio quedaron definidas como programación neurolingüística, sustentada por Goleman, Grinder y Blender (2015), (Echeverría, 2014),

1 Jhon Arjona De León, estudiante de pregrado 10 semestre de Licenciatura en Matemática y Física, Universidad Popular del Cesar.jarjona@unicesar.edu.co

2 Eva De la Hoz Contreras, estudiante de pregrado 10 semestre de Licenciatura en ciencias Naturales y Medio Ambiente, Universidad Popular del Cesar. esandrithdelahoz@unicesar.edu.co

3 Dra. Maria Mercedes Colinas, docente Universidad Mariana. mercede3371@gmail.com

4 Dr. Teovaldo García Romero, docente Universidad Popular del Cesar, categoría Titular, investigador Senior, líder grupo de investigación interdisciplinario pensamiento numérico, políticas públicas de ciencia, tecnología e innovación, medio ambiente, problemas de la educación Latinoamericana y del Caribe, categoría A, según Minciencias. teovaldogarcia@unicesar. edu.co Orcid Id https://orcid.org/0000-0002-6398-5113. 
y desempeño académico por Edel (2012), Torres, (2010), De Giraldo (2013). El proyecto está soportado por el paradigma positivista, con diseño no experimental, de campo descriptivo transaccional en su dimensión temporal; por su naturaleza cuantitativa lo lleva al proceso de codificación, tabulación, y análisis estadístico; para ello se toma como población (8) docentes y 17 estudiantes de la facultad de ciencias básicas y de educación, considerando desde el primer semestre hasta el quinto; se les aplico un cuestionario tipo Likert con escala de cuatro alternativas de respuesta, para medir las variables propuestas. El análisis de los resultados permitió establecer estrategias para el fortalecimiento de las habilidades estudiantiles desde el comienzo de la carrera apoyándose en el desarrollo de competencias sobre la programación neurolingüística, aumentando el compromiso y responsabilidad social como futuros licenciados del país.

\section{PALABRAS CLAVES:}

programación neurolingüística, desempeño académico, contexto institucional

\section{ABSTRACT}

The objective of the research is to analyze neurolinguistic programming as a strategy in the academic performance of students of the Faculty of Basic Sciences and Education. The study variables were defined as neurolinguistic programming, supported by Goleman, Grinder and Blender (2015), (Echeveria, 2014), and academic performance by Edel (2012), Torres, (2010), De Giraldo (2013). The project is supported by the positivist paradigm, with a non-experimental design, a transactional descriptive field in its temporal dimension; Due to its quantitative nature, it leads to the process of coding, tabulation, and statistical analysis; For this, the population is taken as (8) teachers and 17 students from the Faculty of Basic Sciences and Education, considering from the first semester to the fifth; A Lickert-type questionnaire with a scale of four response alternatives was applied to them to measure the proposed variables. The analysis of the results made it possible to establish strategies to strengthen student skills from the beginning of the career, relying on the development of competencies on neurolinguistic programming, increasing commitment and social responsibility as future graduates of the country.

\section{KEYWORDS:}

neurolinguistic programming, academic performance, institutional context

\section{INTRODUCCIÓN}

El contexto universitario es referencia para la evolución de los pueblos, sin embargo, en el área académica el esfuerzo de los estudiantes no les garantiza el éxito, son las destrezas $y$ habilidades las que comienzan a tomar fuerza en su desempeño, pues este se ve condicionado por la capacidad cognitiva que les permite elaborar representaciones mentales con implicaciones causales en el comportamiento. Esta forma de ver lo que sucede, simboliza un elemento determinante para la construcción de los saberes; de acuerdo con el modelo constructivista y cognoscitivo, en función que percibirse como hábil es el eje central para conseguir el significado del mismo.

Cabe acotar, que el siglo XXI se ha caracterizado por tener los cambios más acelerados de la historia; en este sentido, la UNESCO (2005) promulga que la educación superior debe orientarse primero hacia un sentido social que integre el desarrollo del individuo con el entorno y segundo resaltar las bases del conocimiento sobre competencias requeridas en el área laboral, considerando la alineación del aprendizajes del estudiante con el desarrollo cultural y social que orienta su formación, con base al desafío que requiere el campo profesional, este proceso de evolución trae 
consigo la necesidad de modificar algunos esquemas tradicionales que definitivamente ya no satisfacen las necesidades del entorno.

Desde este punto de vista, la programación neurolingüística como estrategia que permite elaborar un conjunto sistemático de operaciones puede ser utilizada tanto por el docente como por el estudiante para fortalecer el desempeño académico, puesto que implica un proceso que sintoniza las necesidades reales del sistema con los estilo de aprendizaje individuales, reforzando la comunicación asertiva, así como los conocimiento sobre competencias requeridas en el contexto, entre otros aspectos; de tal manera, que en el acto pedagógico se produzca una experiencia significativa impulsando las habilidades con la práctica.

Bajo este panorama, el contexto académico latinoamericano revela que el enfoque de la enseñanza universitaria persiste en mantener estrategias que conllevan a un aprendizaje mecánico, anclada en la transmisión de datos y conocimientos poco relevantes para su aplicación en la vida cotidiana, por ello las conductas de los estudiantes son el reflejo de las actitudes de los docentes, de allí la necesidad de hacer consciencia que la forma como se enseña puede modificar el aprendizaje, impulsando nuevos comportamientos motivados hacia la gestión efectiva del conocimiento, la calidad de producción académica y por ende el desarrollo de competencias profesionales.

Colombia, no escapa de esta realidad, los resultados de las pruebas PISA (2012), han quedado por debajo de la media a nivel internacional, de igual manera sucede con las pruebas SABER realizadas internamente en el país, en las cuales se consideran la evaluación de la lectura crítica, resolución de problemas, obviando y la forma como se utilizan los conceptos científicos en la vida cotidiana, dejando a un lado el aspecto afectivo y comunicativo donde se enmarca lo anterior.
Esta situación se refleja en los escenarios de la Universidad Popular del Cesar, a través de observaciones no estructuradas se evidenció que los docentes en su acción pedagógica carecen de estrategias que orienten a los estudiantes a verificar el cómo se aprende, alejándose de sus necesidades reales; situación que dificulta el reconocimiento de los canales de representación que se utilizan para apropiarse de un aprendizaje significativo, originando un desempeño por debajo de lo esperado, ausencia de motivación, falta de gestión emocional, habilidades sociales pocas desarrolladas, entre otras, generando un efecto domino en la comunicación de las partes involucradas, convirtiendo el espacio de intercambio de saberes en un lugar tenso y llenos de dudas con dificultades en los acuerdos, notándose una visión con escasa proyección creativa para potenciar las competencias requeridas sobre el dominio del saber específico.

En este orden de ideas, aun cuando las habilidades y el esfuerzo de los estudiantes se hacen presente en su desarrollo académico, su desempeño muestra falta de motivación, hábitos de estudios mal formados, baja autoestima; elementos que interfieren en el desarrollo de sus competencias, presentando indicadores de frustración en el aprendizaje e incluso deserción académica.

Esta problemática, sienta la necesidad de adentrarse a nuevas formas de enseñar $y$ aprender desde la programación neurolingüística como estrategia para fortalecer el desempeño académico, permitiéndole al estudiante y al docente apropiarse de sus destrezas, así como mejorar la forma de actuar y comunicar en coherencia con las necesidades presentadas, soltando los tradicionales esquemas de trabajo para alinearse a los desafíos educativos que exige la época.

De continuar esta situación, tanto los docentes como los estudiantes seguirán enmarcados 
en antiguos paradigmas, orientados solo conocimiento específico de un área, distantes de un desarrollo integral que conlleve a comprender el significado de lo aprendido, alejándose de la oportunidad de apropiarse de sus competencias para enfrentar los desafíos como profesional, sin la posibilidad de encontrar dentro del aula las respuestas para abrazar la incertidumbre y poder lograr los objetivos propuestos. Por lo expuesto, la problemática es abordada desde el análisis de la programación neurolingüística en el desempeño académico de los estudiantes de la facultad de ciencias básicas y de la educación de la UPC.

En consonancia con lo anterior, la programación neurolingüística, aun cuando ha sido poco aplicada por docentes dentro del aula como estrategia que potencia el proceso de enseñanza aprendizaje, es clave para gestionar una comunicación de calidad, descubrir las destrezas individuales y colaborativas de un grupo, así como fortalecer el pensamiento crítico y creativo, base para aumentar la autoestima y liderar los propios procesos de aprendizaje.

De allí que, se requiere tomar consciencia que la esencia del acto académico esta mediada por la comunicación; a partir de allí, se gestan las relaciones lo cual permite el acercamiento entre los docentes y estudiantes generando un clima de confianza reciproco que afecta de manera negativa o positiva el comportamiento de ambos actores y del sistema en general.

En este sentido, para innovar en los procesos de enseñanza aprendizaje se plantea como objetivo general analizar la programación neurolingüística en el desempeño académico de los estudiantes de la facultad de ciencias básicas y de la educación de la UPC y como objetivos específicos:

Identificar los elementos que intervienen en la programación neurolingüística como estrategia en el desempeño académico de los estudiantes de la facultad de ciencias básicas y educación.

Describir los factores internos que intervienen en el desarrollo del desempeño académico de los estudiantes de la facultad de ciencias básicas y educación.

Caracterizar los elementos externos que intervienen en el desempeño académico de los estudiantes de la facultad de ciencias básicas y educación.

Proponer estrategias de programación neurolingüística que fortalezcan el desempeño académico de los estudiantes de la facultad de ciencias básicas y educación.

\section{CUERPO Y DESARROLLO}

\section{PROGRAMACIÓN NEUROLINGÜÍSTICA.}

También conocida como la PNL, es un conjunto sistemático de operaciones centrada en el análisis de la comunicación y de los procesos de cambio. Grinder y Blender (2015), la programación es la capacidad de organizar las percepciones que se tienen del medio a través de los sentidos, neuro significa funcionamiento de la totalidad del cerebro para jerarquizar lo que se percibe, y lingüística representa la forma de expresar lo percibido verbal y no verbal, en conjunto resultan en una forma de comunicación particular; es decir todo comportamiento proviene de procesos neurológicos, mediado por el lenguaje. enfocando el aprendizaje desde el constructivismo con resultados extraordinarios en cualquier ámbito de la experiencia humana.

Siguiendo el orden, (O'Connor y Seymour, 2014), la definen como una herramienta cognitiva, mediada por el lenguaje, que influencia y persuade; puede ser utilizada en los espacios educativos para diagnosticar las necesidades, planificar en función de ellas y escoger estrategias cónsonas a la realidad. También para comprender que los procesos de 
comunicación dirigen los pensamientos logrando los objetivos propuestos; por ello, detrás de un conocimiento teórico debe estar inmerso el componente procedimental con relevancia en la acción y en el área afectiva, razón que explica la importancia de la PNL en los procesos de desempeño académico en los estudiantes. Por ello, no basta con tener una capacidad intelectual alta, el éxito depende de la interpretación que se les otorgue a las experiencias educativas.

\section{ELEMENTOS DE LA PROGRAMACIÓN NEUROLINGÜÍSTICA.}

En la programación neurolingüística, como modelo de comunicación que apoya al rendimiento académico, intervienen varios elementos que la constituyen y le dan forma, tales como: las creencias, empatía, flexibilidad y congruencia, con el propósito de mejorar las relaciones intrapersonales e interpersonales, desde cualquier espacio relacional.

Creencias o presuposiciones. Forman parte de la estructura más profunda del pensamiento. Para (Echeverría, 2014), son juicios, que hacen parte del pasado y se reflejan en las actitudes, valores o ideologías del presente, afectando los espacios del futuro; es decir, lo que se piensa hoy es el reflejo de lo que sucederá mañana; lo cierto es que aun cuando están presentes para proteger, proveer y prevenir al individuo, en algunas oportunidades dejan de ser funcionales para vivir en la realidad que se conoce. Por su parte, (Martínez, 2005) afirma que las creencias representan los pensamientos que acompañan al individuo para actuar de una manera determinada, considerándolas como verdaderas sin tener pruebas objetivas que las respalden; sin embargo, obstaculizan o facilitan la construcción del conocimiento; de allí, que la interpretación de lo que se vive es válida para cada quien.
Empatía. Es la capacidad que tiene el individuo de ponerse en el lugar del otro, percibiendo su emocionalidad. (Drucker, 2007). Esta se refleja a través del lenguaje corporal, está determinada por la escucha y el discurso, su clave subyace en saber sintonizar la información con las sensaciones, sentimientos, valoraciones, intenciones y acciones reflejadas en el comportamiento.

Al respecto, Goleman (2014), alude que el termino de empatía se utiliza para valorar los procesos internos de las personas. En este sentido, cuando un docente toma consciencia de su desempeño como líder, tiene la capacidad de comprender lo que le sucede a sus estudiantes y diseñar estrategias con herramientas que favorezcan al colectivo alineándose a sus necesidades, así como a los desafíos del entorno para apoyar el éxito académico de los mismos.

En efecto, cuando el docente como modelador de procesos de aprendizaje es empático, abre el espacio de confianza que le permite ocuparse la emocionalidad y afectividad; por consiguiente; los contenidos compartidos en el quehacer pedagógico le otorgan significado al aprendizaje. Esta relación facilita la contextualización de los temas con la realidad de cada estudiante, permitiendo que intervengan y reflexionen sobre lo que acontece; lo cual afianza la seguridad en el desempeño académico, las destrezas, el manejo de las responsabilidades y refuerza la disposición para modificar las conductas ajustándose a los nuevos desafíos.

Flexibilidad. Para Echeverría (2014), es la capacidad, condicionada por las creencias, de desarrollar procesos de manera eficiente ante los estímulos externos; cuando el docente asume la flexibilidad como parte del proceso de enseñanza, puede centrar sus estrategias en la particularidad de los estudiantes ofreciendo recursos apropiados, e incluso darle paso a la utilización de la tecnología disminuyendo los 
traumas, generando un espacio de confianza en sus competencias, así como en las capacidades de los estudiantes, ampliando la visión del quehacer pedagógico y se enriqueciendo la forma de aprender, lo cual origina la construcción de nuevos significados, cambiando el modo particular de actuar.

Congruencia. Esta referida a la capacidad que tiene el individuo de adaptarse al entorno en coherencia con los pensamientos, sentimientos y acciones. En palabras de Maturana (2013) "todo sistema existe solo para la conservación de su adaptación y organización", esto sucede porque los comportamientos van solo hacia un objetivo, y cuando existe la consciencia suelen reducirse los conflictos internos; en este sentido, como elemento de la programación neurolingüística, la congruencia está orientado a definir las estrategias a corto y mediano plazo con un valor constante asumiendo la relación con el medio la cual considera variaciones validas en el proceso.

Bajo estas interpretaciones, los elementos de la programación neurolingüística representan los aspectos del comportamiento del docente y del estudiante en su práctica educativa, los cuales encausan el accionar sobre las experiencias; una vez que se hacen conscientes revelan la capacidad de modificar aquellas estrategias que ya no funcionan, flexibilizando la forma y el fondo de las actividades utilizadas para fortalecer en rendimiento académico con efectos directos en la comunicación en los actores del proceso. (Kaplan y Norton 2004).

\subsubsection{Desempeño académico. Para Pérez.} (2005) es la suma de diferentes y complejos factores que influyen en la persona que aprende, $y$ ha sido determinado como un importe atribuido al logro del estudiante en las tareas académicas, el autor afirma que se puede calcular a través de las calificaciones obtenidas, con una valoración cuantitativa, cuyos resultados muestran las materias ganadas o perdidas, la deserción y el grado de triunfo académico, para los estudiantes universitarios constituye un elemento imprescindible para la valorar la calidad educativa en la enseñanza superior..

Por otro lado, Torres (2010), plantea que el desempeño académico de los estudiantes está determinado en gran medida por sus historias familiares, características individuales, educativos y su compromiso por alcanzar las metas que se plantean como proyecto de vida. Desde esta perspectiva, se considera como un fenómeno educativo de carácter complejo e integral que involucra tanto a los estudiantes con sus logros y experiencias en su interacción con la educación superior, como a los diversos elementos institucionales que contribuyen a la iniciación profesional y formación integral de estos como sujetos pensantes y críticos;

En este sentido, para que el desempeño académico sea satisfactorio se requiere la utilización de estrategias enfocadas en la programaciónneurolingüísticaquefortalezcanlos procesos formativos liderados por los docentes, enrutando el aprendizaje de manera sistémica, a través de una comunicación bidireccional, donde se consideren la transformación de las creencias con flexibilidad y congruencia en el quehacer educativo, integrando las competencias del conocer, hacer, ser y convivir coadyuvantes en la responsabilidad de los actores y el efecto sobre el entorno.

\section{DESEMPEÑO ACADÉMICO}

Para Vivas, Vásconez y Vivas (2019), es conjunto de factores que interviene en el aprendizaje de los estudiantes para adquirir competencias tanto a nivel cognitivo como procedimental, las cuales evidencian la forma como enfrenta la realidad y se convierten en determinantes para el saber especifico a la hora de ejercer su profesión.

Por su parte Díaz et al, (2017), afirma que el desempeño académico está directamente 
relacionado con la capacidad de respuesta del estudiante, donde pone de manifiesto el aprendizaje que tiene en su saber; aun cuando está condicionada por factores propios internos y externos, el resultado depende directamente de la interpretación del mismo y como se enfrente a las experiencias que vive en el entorno.

En este orden, la tarea de los docentes de la educación superior es formar al estudiante en presente para el futuro con fortaleciendo sus capacidades, de tal mera que puedan hacer consciencia de su responsabilidad tanto en el área académica como en lo profesional, de allí, al integrar en su desempeño herramientas de programación neurolingüística logra conseguir el significado del para que, tomando sus propios recursos para desafiar las exigencias del entorno.

\section{FACTORES INTERNOS QUE INTERVIENEN EN EL DESARROLLO DEL DESEMPEÑO ACADÉMICO.}

Los factores internos y externos que intervienen en el desempeño académico, pueden ser multicausales, puesto que son consecuencia de la interacción del estudiante con su propio proceso de desarrollo y con el medio donde se desenvuelve, esta situación modifica la percepción, por tanto, el comportamiento y el posible efecto de los resultados que se quieran alcanzar; es decir estos condicionantes van a depender de las experiencias particulares de cada sujeto (Benítez, Giménez y Osicka, 2000).

Por su parte, Jiménez (2000) refiere que se puede tener capacidad intelectual y actitudes en el abordaje del desempeño académico, sin embargo, no es garantía de tener un rendimiento acorde a lo esperado, en este orden considera:

Motivación. Es el producto de dos fuerzas principales que mueven al individuo, la expectativa de alcanzar una meta y el valor que tiene para él; en otras palabras, el estudiante requiere trabajar hacia un objetivo; además necesita un esfuerzo adicional en sus acciones para lograr el éxito. Jiménez (2000). Al respecto, Alcalay (2007) involucra variables cognitivas como las habilidades de pensamiento y las conductas instrumentales para alcanzar las metas propuestas, así como también las afectivas, las cuales comprenden elementos como la autovaloración, autoconcepto, ambas actúan en interacción a fin de complementarse y hacer eficiente la motivación. Este panorama conlleva a develar que la motivación es factor clave en el desempeño académico, considerando que es el motor que mueve al individuo antes, durante y después del proceso de enseñanza.

Autocontrol. Es la habilidad que tiene el estudiante para regular los impulsos, emociones, deseos y acciones, está relacionado con un conjunto de prácticas que pueden aprenderse y desarrollarse con la experiencia, entre ellas el reconocimiento de los canales de representación sensoriales que permiten la percepción e interpretación de la experiencia.

Cuando el estudiante gestiona su autocontrol, mantiene un comportamiento adecuado en el entorno educativo universitario, laboral, social, entre otros; si por el contrario no son gestionados con asertividad, conllevan a cometer errores de los cuales habrá un arrepentimiento dejando marcas en la personalidad. Casanova (2002).

En este sentido, se ha encontrado que los individuos con más altas calificaciones poseen autocontrol, lo cual interviene favorablemente al utilizar su inteligencia emocional para solucionar alguna situación en particular. Woolfolk (2006), resaltando las atribuciones que hacen las personas sobre sí mismas y sobre los demás.

Por lo tanto, para aumentar el desempeño académico del estudiante se requiere que el docente también gestione su autocontrol permitiendo interactuar con el mismo a través de la comunicación, de tal manera que pueda 
conocer sus necesidades, y partir de allí para fortalecer las competencias, motivación, perseverancia, empatía, y la agilidad mental; configurando los rasgos de carácter como la autodisciplina, la compasión ó el altruismo, que resultan indispensables para el desempeño profesional.

Competencia cognitiva. Es la capacidad que tiene estudiante de procesar una información, donde intervienen los procesos cognitivos inferiores y superiores para luego adaptarlos a los diferentes espacios donde se desenvuelva. Martínez, (2005) lo define como las condiciones cognitivas del aprendizaje significativo. En este sentido, las competencias cognitivas se manifiestan directamente en las capacidades intelectuales que resultan de la disposición que muestran los individuos al hacer algo. Por lo tanto, se hace necesario incorporar técnicas y estrategias basadas en la programación neurolingüística que posibiliten la utilización de las habilidades cognitivas desde un aprendizaje significativo.

Inteligencia. Es un constructo utilizado para estimar, explicar o evaluar algunas diferencias conductuales entre las personas: éxitos / fracasos académicos, modos de relacionarse con los demás, proyecciones de proyectos de vida, desarrollo de talentos, notas educativas, resultados de test cognitivos, entre otras, (Pimienta, 2008). En este orden, la inteligencia emocional es una forma de interactuar con el mundo que tiene muy en cuenta los sentimientos, y engloba habilidades tales como el control de impulsos, la autoconciencia, entre otros, configurando rasgos de carácter que resultan indispensables para una buena y creativa adaptación social. Goleman (2014) La inteligencia es un buen predictor de los resultados académicos, lo cual produce una relación significativa entre el individuo y el medio.

\section{ELEMENTOS EXTERNOS QUE INTERVIENEN EN DESARROLLO DEL DESEMPEÑO ACADÉMICO.}

1.2.2.1 Hábito de Estudio. García (2000), afirma que los hábitos de estudio se establecen con la repetición del acto de estudiar bajo condiciones ambientales de espacio y tiempo con características iguales, como primer paso para activar y desarrollar la capacidad de aprender. Para Ferrer (2007) tienen la finalidad de lograr un aprendizaje, entendido este desde una postura cognitiva como un proceso de comprensión, integración e interacción entre el sujeto y el medio, que conlleva a la asimilación, acomodación y reproducción de lo aprendido; en este sentido el estudiante tiene la capacidad de percibir, pensar, analizar y relacionar las ideas logrando la comprensión para el aprendizaje.

Cabe destacar que una de las principales causas del bajo desempeño académico se focaliza en los hábitos inadecuados para estudiar desde un nivel básico hasta el universitario, problema que al mismo tiempo genera dificultades de aprendizaje que van más allá de lo estadístico, reflejando deficiencias en la calidad educativa que se denota a través de las pruebas ICFES, con un bajo porcentual según lo esperado para el desempeño; situación que repercute en el ámbito profesional una vez que egresan los estudiantes del sistema universitario. Tovar, (2003).

Por lo tanto, el docente requiere incluir estrategias innovadoras de programación neurolingüística dentro del aula con prácticas dirigidas, para que los estudiantes puedan contactar con los beneficios que genera la adquisición de hábitos y como estos a través de la disciplina pueden llevarlos hacia el éxito académico y profesional.

Contexto Socioeconómico. Seibold (2007), señala que la correlación entre el aprendizaje y el contexto socioeconómico, puede ser causal del éxito o fracaso académico; sin embargo, 
si bien es cierto el contexto socioeconómico afecta el nivel de calidad educativa, este no lo determinan, por ello se requiere realizar una observación específica a modo de verificar su influencia con mayor exactitud. Para Cohen (2008) el contexto socioeconómico que vive el estudiante influye y condiciona el proceso de aprendizaje del mismo. Por lo tanto, el docente debe investigar sobre el estatus del estudiante de tal manera que pueda considerar su situación a la hora de realizar las actividades pedagógicas.

Contexto Institucional. Según Loaiza y Duque (2004), los factores institucionales marcan un precedente sobre el rendimiento académico; entre ellos se pueden destacar variables como: los horarios de los cursos, tamaños de grupos o criterios de ingreso en carrera, así como aspectos pedagógicos, que inclusive pueden estar relacionados o entrelazarse con elementos personales y sociales. En este orden, el ajuste con la situación real y la deseada dejan ver si los estudiantes están satisfechos con su elección profesional.

\section{ESTRATEGIAS DE PROGRAMACIÓN NEUROLINGÜÍSTICA EN EL DESEMPEÑO ACADÉMICO.}

En los términos de PNL, la estrategia va más allá del camino que lleva a lograr las metas propuestas. Es disponer del funcionamiento del sistema neurológico de manera integrada al comportamiento; de tal forma, que se puedan hacer conscientes los procesos mentales para generar respuestas visuales, auditivas, y kinestésicos acordes a las exigencias educativas y sociales. Por ello se recomiendan las siguientes estrategias que apoyan el desempeño académico del estudiante:

Anclar las capacidades de los estudiantes a través de estímulos externos sensoriales, asociados a sus conductas como olores, sabores, palabras o gestos que los lleve a evocar y registrar en la memoria los pasajes previos relacionados con el conocimiento, de tal manera que puedan retener la información.

Reencuadrar el marco de referencia del contenido, para cambiar el significado de los hechos, así los estudiantes cambian el estado emocional, es decir utilizar chistes, anécdotas o hechos inesperados referidos al tema, incentivando a realizar actividades creativas como valor intangible al éxito.

Utilizar las submodalidades, a través de la imaginación jerarquizando y clasificando para decodificar las experiencias para modificar las situaciones de aprendizaje que fueron desagradables.

Hacer pausas activas de relajación para equilibrar el pensamiento y lograr las metas propuestas, considerando la dimensión muscular, la ampliación de la consciencia y la liberación del espíritu.

Calibrar conociendo en forma precisa el comportamiento de los estudiantes, realizando actividades innovadoras conducentes a despertar la consciencia de las destrezas y puedan apropiarse de su saber específico de manera significativa.

Estas estrategias, convergen en los sistemas de creencias y la forma de comunicación que establecen los docentes y los estudiantes, para mejorar de manera continua su propio comportamiento, asumiendo la responsabilidad que cada una de las partes tiene en el proceso educativo.

\section{METODOLOGÍA}

\subsection{Postura epistemológica}

La investigación se enmarca dentro del paradigma cuantitativo, con un enfoque empírico positivista. Hurtado (2002); utiliza la lógica de la verificación empírica de los hechos y sus causas, la complejidad del ser humano 
reducida a variables, calcula la probabilidad estadística; además, se aplican instrumentos que codifican, tabulan y analizan para concretar las conclusiones.

\subsection{Tipo de Investigación}

El tipo de investigación se encuadra dentro del enfoque analítico, descriptivo de campo, el cual Hernández, Fernández y Batista (2012, p103), plantean que los estudios descriptivos pretenden medir información de manera independiente o conjunta sobre la variable objeto de estudio, ofreciendo la posibilidad de predicciones o relaciones, aunque sean poco elaborada. En este tipo

de investigación se reinterpreta lo analizado en función de ciertos criterios seleccionados por el investigador, intentando identificar las sinergias menos evidentes de los eventos presentados; en este caso, se estudian los elementos de la programación neurolingüística, los elementos que intervienen en el desempeño académico para proponer estrategias que fortalezcan la función docente y las competencias de los estudiantes.

\subsection{Diseño de la investigación.}

El estudio se ha desarrollado utilizando un diseño de campo, no experimental transeccional descriptivo en su dimensión temporal, recolectando los datos en un solo momento para describir las variables de investigación y medir su incidencia en ese momento. empleando para ello la revisión documental de registros institucionales y el cuestionario estructurado como técnica de recopilación de información.

\subsection{Población de Estudio}

Parra (2000), expone que la población es el conjunto de todos los elementos, seres u objetos que contienen las características y mediciones $\mathrm{u}$ observaciones que se requieren en una investigación dada. Para tal fin, la población se selecciona basada en un conjunto de características homogéneas o específicas según el criterio del investigador, estas especificaciones son: docentes pertenecientes a la facultad de ciencias básicas y educación, docentes del turno diurno, docentes pertenecientes a la Universidad Popular del Cesar, consiguiendo la colaboración de ocho (8) docentes en los semestres del quinto al octavo pertenecientes a la misma universidad en de la facultad de ciencias básicas de educación de los programas de matemática y ciencias naturales. Igualmente, se consideraron diez y siete (17) estudiantes inscritos en la facultad de ciencias básicas y de educación de la Universidad Popular del Cesar, estudiantes inscritos en los semestres desde el quinto hasta el octavo periodo 2020 de la faculta de ciencias básicas y de educación, del turno diurno prestos a apoyar la investigación.

\subsection{Técnicas e Instrumento de recolección de datos}

Para efectos de esta investigación, se considera utilizar información no confidencial disponible sobre las variables de estudio en la Universidad Popular del Cesar, así como en otros registros institucionales, de igual manera la técnica a aplicar es la encuesta en su modalidad cuestionario.

Ahora bien, en esta investigación se construye un cuestionario tipo Likert, el cual según Hernández, Fernández y Baptista (2006), se configura por afirmaciones ante las cuales el encuestado emite su opinión en grados de intensidad. En este contexto, la investigación presenta un Instrumento de recolección de información para docentes y otro para estudiantes, los cuales recogen en total 54 ítems cerrados y medidos a través de una escala de alternativa fija de repuesta. Con base al tipo de escala se asumió la siguiente ponderación para la medición de las repuestas: (4) Siempre, (3) Casi siempre, (2) Casi Nunca, (1) Nunca. A continuación, se 
muestra el cuadro \#1 identificando como ponderaciones de ítems del cuestionario.

\section{Cuadro \# 1}

Baremo para la interpretación de la media.

\begin{tabular}{|l|l|l|l|}
\hline RANGO & ALTERNATIVA & INTERVALOS/LÍMITES & CATEGORÍA \\
\hline 4 & Siempre & $3,28 \leq X \leq 4,00$ & Alta \\
\hline 3 & Casi Siempre & $2,52 \leq X \leq 3,27$ & Medianamente Alta \\
\hline 2 & Casi Nunca & $1,76 \leq X \leq 2,51$ & Baja \\
\hline 1 & Nunca & $1,00 \leq X \leq 1,75$ & Muy Baja \\
\hline
\end{tabular}

Fuente: Arjona, De la Hoz, García y Colina (2020)

De igual modo, se presenta a continuación un cuadro correspondiente al rango, intervalo, categoría y descripción que se tomará en cuenta para el análisis de la desviación estándar de los resultados, alcanzando con ello realizar afirmaciones sobre la confiabilidad de la información obtenida en la aplicación del instrumento de recolección de datos.

\section{Cuadro \#2}

Baremo para la Desviación Estándar

\begin{tabular}{|l|l|l|l|}
\hline Rango & Intervalo & Categoría & Descripción \\
\hline 1 & $4.50-6.00$ & Alta Dispersión & $\begin{array}{l}\text { La categoría indica una baja confiabilidad } \\
\text { en el nivel de compromiso }\end{array}$ \\
\hline 2 & $3.00-4.49$ & $\begin{array}{l}\text { M o d e r a d a } \\
\text { Dispersión }\end{array}$ & $\begin{array}{l}\text { La categoría indica una moderada } \\
\text { confiabilidad en el nivel de compromiso }\end{array}$ \\
\hline 3 & $1.50-2.99$ & Baja Dispersión & $\begin{array}{l}\text { La categoría indica una alta confiabilidad } \\
\text { en el nivel de compromiso }\end{array}$ \\
\hline 4 & $0.00-1.49$ & Muy Baja Dispersión & $\begin{array}{l}\text { La categoría indica una muy alta } \\
\text { confiabilidad en el nivel de compromiso. }\end{array}$ \\
\hline
\end{tabular}

Fuente: Arjona, De la Hoz, García y Colina (2020)

\section{TÉCNICA DE ANÁLISIS DE LA INFORMACIÓN}

Para llevar a cabo el análisis de la información, se procedió en primer término a la codificación y tabulación de los datos. Al respecto, Tamayo y Tamayo (2006), indican que la codificación y fabulación es una parte del proceso de análisis de datos, permite la organización y recuento de los mismos, determinando el número de los casos que corresponde a cada categoría de respuesta.
En este sentido, la técnica estadística utilizada para esta investigación es descriptiva con medidas de tendencia central, para llevar a cabo el tratamiento de la información el cual permite la descripción de las variables de estudio lo cual según Hernández, Fernández y Baptista (2006), específicamente se realiza a través del uso de las técnicas de medidas de tendencia central.

Dentro del mismo contexto, se utilizó la media aritmética, la moda y la mediana que permite 
la categorización de ítems, indicadores, dimensiones, así como de las variables de estudio, en relación a análisis los procesos relacionados a los dominios del proceso de liderazgo resonante.

\section{RESULTADOS DE LA INVESTIGACIÓN}

Una vez aplicado el cuestionario a los informantes identificados en un número de 40 docentes y 200 estudiantes, se obtuvo una matriz de datos, procesada a través del paquete estadístico Excel 2010, para el análisis de los mismos. La presentación de los resultados para cada variable y grupo de dimensiones e indicadores, se hacen a través de la estadística descriptiva, con el cálculo de medidas de tendencia central.

Variable: Programación neurolingüística. № 8 Docentes

Dimensión: Elementos de neurolingüística

\section{Indicador: Creencias.}

El indicador creencias, muestra un promedio de 2,43 ubicándose en categoría baja y una desviación estándar de 1,06 señalando que la información encontrada es confiable por su muy baja dispersión; evidenciando que tanto el $19,17 \%$ y el $28,33 \%$ de los informantes docentes expresan que siempre y casi siempre, respectivamente, consideran que sus conocimientos versan sobre lo aprendido anteriormente, las cuales pueden llegar a influir negativamente en su gestión. En este sentido, el $28,33 \%$ de expresan que casi nunca verifican los canales de aprendizaje de lo estudiante para establecer sus estrategias, mientras, que el $24,17 \%$ consideran hacer un diagnóstico previo.

\section{Indicador. Empatía}

Los resultados muestran la empatía con un promedio de 2,46 el cual lo ubica en la categoría Baja, donde el 21,67 y el $25,83 \%$ de los encuestados manifiestan que siempre y casi siempre buscan comunicarse con sus estudiantes para gestionar su aprendizaje, el $29,17 \%$, casi nunca consideran los canales de comunicación para cumplir con el rol de asesor, mientras que solo el $23,33 \%$, expresan que nunca consideran su comunicación en función de las necesidades presentadas por los estudiantes

\section{Indicador: flexibilidad}

Se evidencia un promedio de 2,37 ubicándolo en la categoría Baja con una desviación estándar de 1,04 señalando que la información encontrada es de muy alta confiabilidad por su baja dispersión, en consecuencia el $25,83 \%$ de los informantes de la facultad de ciencias básicas y educación logran casi siempre flexibilidad en su forma de llevar su accionar pedagógico, mientras el 17, 50 $\%$ afirma que siempre es flexible en la utilización de estrategias para mejorar el rendimiento, el $32,50 \%$, asume que casi nunca emitir mensajes cónsonos con lo que sienten, y el 24,17 que nunca emite mensajes que no estén acordes a lo que se quiere transmitir.

\section{Indicador: congruencia}

Se muestra en una categoría baja por presentar una media de 2, 24 con una desviación estándar de 1,04 marcando una alta confiabilidad. Al respecto, se refleja que el $29,38 \%$ manifiesta casi nunca utilizan las estrategias de programación neurolingüísticas coordinadas con los intereses de los estudiantes, mientras que el $25,63 \%$ opinan que casi siempre cumple con su programación gestionando su forma de hacer docencia. En este sentido, se destaca que el $30,62 \%$, consideran que nunca realiza ejercicios que estén fuera de los contenidos desarrollados en clase y solo el $14,38 \%$ de los informantes evidencian la capacidad de alinear, pensamiento, acción y emoción mostrando interés para que los estudiantes logren un desempeño exitoso. 
Variable: Desempeño Académico. $\mathrm{N}=17$ estudiantes

Dimensión: Elementos internos y externos que intervienen en el desempeño académico.

Los resultados de la dimensión de elementos internos arrojaron un promedio de 2,38 ubicándolo en una categoría Baja, con una desviación estándar de 0,08 siendo esta significantemente de muy alta confiabilidad y muy baja dispersión, es decir indica una muy alta confiabilidad en el nivel de compromiso. El indicador motivación refleja un 2,81 de promedio siendo este de moderado dominio indicando que el $37,50 \%$ de los estudiantes opinaron que casi nunca obtienen la suficiente motivación de los docentes para el logro de un desempeño académico eficaz. De igual forma, se observa que el $34,17 \%$ de la población opinaron que casi siempre el docente se dirige hacia el logro de la tarea y el conocimiento pero que en su discurso no se detiene a mirar el estilo de aprendizaje solo se enfoca a cumplir su objetivo, el 25,0 responden que siempre el docente se ocupa de motivarlos y solo el 3,33\% manifiestan que nunca utiliza estrategias para elevar su participación en clase.

Por otro lado, el indicador auto Control, posee un promedio de 2,28 , igualmente es de bajo dominio, aquí se observa que el $41,25 \%$ de los estudiantes mantienen relaciones que hacen modelar su inteligencia permitiendo la oportunidad de fortalecer sus habilidades, motivación, entusiasmo, perseverancia, empatía, y la agilidad mental que resultan indispensables para una buena y creativa adaptación social; un $31,88 \%$ afirman que casi nunca gestionan su forma de aprender, el $17,50 \%$ de los encuestados resumen que nunca han tenido que auto controlarse y el 9,38 manifiestan que siempre requieren pensar antes de dirigirse a sus compañeros y al docente.
Seguidamente, para el indicador competencia cognitiva, el cual posee un promedio de 2,37 de bajo dominio, se observa cómo el 32,50\% de la población estudiantil considera que casi siempre lleva a cabo las tareas de selección, organización, y elaboración de actividades que les permita crecer en el conocimiento.

Así mismo, se observa como el indicador inteligencia con promedio 3,27 muestra un alto dominio, observándose que el $66,67 \%$ de la poblacióncasisiempreysiemprerespectivamente posee la capacidad de memorizar contenidos con facilidad englobando habilidades de control de impulsos, autoconsciencia, mientras que el $32,8 \%$ generalmente prefieren aprender a través de la experiencia.

En cuanto a la dimensión elementos externos, esta se observa con un promedio de 2,20 con bajo dominio, revelando el indicador hábitos de estudio con promedio de 2,19 , donde el $30,63 \%$ de la población de estudiantes manifestaron que casi nunca estudian todos los días y solo lo hacen para los parciales, así el 55,01\% responden que siempre y casi siempre respectivamente utilizan la técnica de la repetición para estudiar y aprenderse los temas, solo el $9,03 \%$ indican que no necesitan memorizar.

Por otro lado, en el indicador socio económico se observa un promedio de 2,80 de moderado dominio, donde el $44,17 \%$ de los estudiantes consideran que casi nunca y nunca influye el estrato económico para que ellos sean aplicados y tengan los tiempos de estudios exactos, sin embargo, el 55,83 de los estudiantes afirman que casi siempre y siempre influye el aspecto económico en su rendimiento.

Ahora bien, el indicador contexto institucional, se observa con un promedio de 2.09 de bajo dominio, donde el $66,67 \%$ de los estudiantes indican que la institución no es un elemento que influya en horario y grupos de estudios, mientras que el $33,33 \%$ de los estudiantes expresan 
que la institución no influye en nada porque la decisión de estudiar a tiempo la tienen ellos.

\section{CONCLUSIONES}

En el análisis realizado, se evidenció ausencia de conocimiento por parte de los docentes sobre los elementos de la programación neurolingüística como estrategias que influyen en la enseñanza aprendizaje de los estudiantes para fortalecer sus competencias académicas, produciendo respuestas poco satisfactorias a la hora de aplicar los conocimientos en un sistema distinto al universitario.

En este sentido, se concluye que los elementos de la programación neurolingüística deben ser representativosparael procesodetransformación de la enseñanza y del aprendizaje, haciéndose necesario una comunicación que contemple los canales sensoriales propios de los procesos cognitivos. El desempeño de los ambos actores (docentes y estudiante) tiende a mejorar, aumentando el grado de empatía y cooperación de los mismos.

Basado en ese escenario, se identifican que los elementos de la programación neurolingüística tienen relevancia en el diseño de estrategias innovadoras, pues permiten abordar las situaciones con coherencia, respondiendo a los desafíos de forma oportuna, lo cual conlleva a la presencia de esquemas de comportamiento con que confluyan en ganar.

En cuanto a los factores internos que interviene en el desempeño académico, se pudo constatar que existe un mejor manejo de la inteligencia en la utilización de estrategias cognitivas, quedando por debajo la motivación y el reflejo del autocontrol, por lo que interfiere en el aprendizaje significativo.

Mientras que, los elementos externos arrojaron que los hábitos de estudios, el contexto socio económico y el institucional también influyen en el desempeño académico, sin embargo, no son determinantes.

Por lo anterior, se requiere que los docentes a través de la programación neurolingüística, impulsen la motivación del estudiante generando estrategias que incluyan los elementos de la programación neurolingüística con fin de fortalecer el rendimiento académico en función de una necesidad real del entorno, para que el aprendizaje perdure en el tiempo de manera significativa y se pueda trasladar al campo profesional.

Se recomienda, realizar entrenamientos a los docentes y a los estudiantes desde cada programa de la Facultad de Ciencias básicas y educación con referencia a los alcances de la programación neurolingüística en el proceso de enseñanza aprendizaje.

Realizar encuentros para diseñar y aplicar las herramientas de la programación neurolingüísticas como espacios de intercambios pedagógicos conducentes a mejorar el rendimiento académico de los estudiantes.

Para los estudiantes, realizar entrenamientos que les permita utilizar las herramientas de la programación neurolingüística para la regulación del autoaprendizaje.

En general, realizar un estudio comparativo con un programa de programación neurolingüística como apoyo al rendimiento académico que muestre el efecto antes y después del programa.

Compartir los hallazgos de este trabajo investigativo para reforzar las competencias académicas y profesionales de los docentes y de los estudiantes. 


\section{REFERENCIAS BIBLIOGRÁFICAS}

Benítez, M; Giménez, M. y Osicka, R. (2000). Las asignaturas pendientes y el rendimiento académico: ¿existe alguna relación?

Casanova (2002). Estrategias docentes para un aprendizaje significativo: una interpretación constructivista. McGraw-Hill.

Cohen, M. (2008). Hábitos de Estudio y Tarea en Casa. Ediciones de la Universidad de Illinois, USA.

Díaz et al, (2017). Rendimiento académico y calidad de vida relacionada con la salud de los estudiantes de odontología. Universidad del Norte.

Druker. (2007). El líder del Futuro. Editorial Deusto.

Echeverría (2014), Escritos sobre Aprendizaje. Comunicaciones Nor-Este. Santiago de Chile.

Edel, R. (2003). Factores asociados al rendimiento académico. Revista Iberoamericana de Educación. Organización de Estados Iberoamericanos para la Educación, la Ciencia y la Cultura. En red. Recuperado.

Edel, R. (2012). El desarrollo de habilidades sociales ¿Determinan el éxito académico? Revista electrónica: Red Científica: Ciencia, Tecnología y Pensamiento.

Ferrer, I. (2007). Clima Organizacional y Rendimiento Estudiantil. Universidad Pedagógica Experimental Libertador. Caracas. Venezuela.

García- Huidobro (2000), Aprendizaje. Ediciones Alfaomega

Goleman (2013); Inteligencia emocional. Editorial Kairós
Goleman, Boyatzis y McKee (2014), Liderazgo, el poder de la inteligencia emocional. Editorial Kairos.

Hernández, Fernández y Batista. (2012). Métodos y técnicas de estudio en la Universidad de Colombia Mc-Graw Hill.

Hurtado (2002). Metodología de la Investigación Holística

Jiménez, M. (2000). Competencia social: intervención preventiva en la escuela. Infancia y Sociedad

Kaplan y Norton (2004). Mapas estratégicos. Como convertir los activos intangibles en resultados tangibles. Gestión 2000.

Loaiza y Duque, (2013). Contexto de las prácticas pedagógicas de los maestros y los docentes. Revista educativa. Universidad de Manizales.

Martínez (2005). Técnicas docentes y sistemas de Evaluación en Educación Superior. Madrid: Narcea

O'Connor y Seymour (2014). Introducción a la programación neurolingüística. Editorial Urano.

Ortiz Rosales (2013). ¿Qué es la programación neurolingüística y cómo funciona? San Luis: Tecnológico de Monterrey.

Parra (2000), Población e investigación. R.R. Bowker,

Pérez. (2005). Desempeño académico. Universidad de Oviedo

Pimienta Prieto, J. H. (2008). Constructivismo: estrategias para aprender a aprender (Tercera ed.). México: Pearson

Sánchez Vázquez, J. (2000). Análisis exploratorio de las variables que condicionan el rendimiento académico. Sevilla, España: Universidad Pablo de Olavide. 
Seibold (2007), Factores asociados al rendimiento académico en estudiantes universitarios, una reflexión desde la calidad de la educación superior pública. Revista Educación 31(1), 43-63, ISSN: 0379-7082

Tamayo y Tamayo (2000). Metodología de la investigación. Limusa. Noriega Editores.

Torres (2010). Bajo rendimiento escolar. INCIPIT. Editores

Tovar. A. (2003). Efectos de los Hábitos de Estudio en el Rendimiento Académico de los Alumnos de la Escuela de Formación Deportiva "Germán Villalobos Bravo". Tesis de Maestría. Universidad Pedagógica Experimental Libertador. Caracas. Venezuela.

Vivas, Vásconez y vivas. (2019). Relación entre los estilos de aprendizaje y el rendimiento académico del estudiantado de la carrera de Ingeniería Agronómica de la Universidad Central del Ecuador. Universidad de Costa Rica.

Woolfolk (2006), Psicología Educativa. Person educación. 